\title{
Norois
}

Environnement, aménagement, société

$234 \mid 2015$

Dynamique des milieux humides, éducation à l'environnement, pratiques festives, tourisme et patrimonialisation

\section{Dynamique de la démographie et du bâti dans l'ouest du Marais poitevin depuis 1705}

Dynamics of building distribution and demography in the western part of the Marais poitevin since 1705

Pierre Pouzet, Axel Creach et Laurent Godet

\section{OpenEdition}

\section{Édition électronique}

URL: https://journals.openedition.org/norois/5589

DOI : $10.4000 /$ norois. 5589

ISBN : 978-2-7535-4218-1

ISSN : $1760-8546$

\section{Éditeur}

Presses universitaires de Rennes

Édition imprimée

Date de publication : 30 juin 2015

Pagination : 83-96

ISBN : 978-2-7535-4183-2

ISSN : 0029-182X

\section{Référence électronique}

Pierre Pouzet, Axel Creach et Laurent Godet, « Dynamique de la démographie et du bâti dans l'ouest du Marais poitevin depuis 1705 », Norois [En ligne], 234 | 2015, mis en ligne le 30 juin 2017, consulté le 13 janvier 2022. URL : http://journals.openedition.org/norois/5589; DOI : https://doi.org/10.4000/ norois.5589 




\title{
Dynamique de la démographie et du bâti dans l'ouest du Marais poitevin depuis 1705
}

\author{
Dynamics of Building Distribution and Demography in the Western Part \\ of the Marais Poitevin since 1705
}

\author{
Pierre Pouzet, Axel Creach, Laurent GodeT*
}

\begin{abstract}
* Auteur correspondant : Tel : +33(0)2 534876 54. Fax : +33 (0)2 53487650
LETG-Nantes-Géolittomer - UMR 6554 CNRS, Université de Nantes, Campus du tertre, BP 81227 - 44312 NANTES

Cedex 3, France (pierre.pouzet@etu.univ-nantes.fr) (axel.creach@univ-nantes.fr) (laurent.godet@univ-nantes.fr)
\end{abstract}

Résumé : Les changements d'usage et d'occupation des sols sont souvent utilisés pour quantifier l'anthropisation des zones humides littorales. Toutefois, la dynamique spatiale et temporelle du bâti et de la démographie sont souvent moins bien prises en compte. A partir de cartes anciennes (1705 et 1820), de photographies aériennes (1950), de la base de données gégraphiques BD-TOPOß de l'Institut Géographique National (IGN) de 2006, ainsi que de recensements communaux de 1801 à 2009, cet article présente la dynamique du bâti et l'évolution démographique de la partie occidentale du Marais poitevin, plus vaste zone humide du littoral atlantique français. D’une manière générale, nous montrons une forte augmentation de la population et des surfaces bâties. Cependant, ces évolutions ne sont régulières ni dans l'espace ni dans le temps. L'évolution des surfaces bâties a surtout concerné la frange littorale et la commune de Luçon et a progressé de manière importante durant la deuxième moitié du xx siècle. L'étude de l'évolution démographique met en lumière deux pics chronologiques : le premier concerne les années 1860 (connu comme le «plein rural ») et le second correspond à la période actuelle. Nous mettons également en évidence le fort essor démographique de la bande littorale. Ces résultats illustrent sur une période historique de trois siècles et avec une résolution spatiale fine des phénomènes observables à l'échelle nationale que sont notamment l'exode rural et la littoralisation.

\begin{abstract}
Land-use and land-cover changes are often used to assess the artificialization of coastal wetlands, but two elements of these major changes are still seldom quantified: the spatial and temporal dynamics of buildings and the demographic evolution. Using old maps and aerial photographs from 1705 to 2006, as well as municipal censuses from 1801 to 2009, we present the dynamics of building distribution and the demographic evolution of the western part of the Marais poitevin, the largest French Atlantic wetland. We show a steep increase in population and in buildings. However, these changes are irregular in both time and space. The increase in building cover mainly occurred in the second half of the $20^{\text {th }}$ century in the littoral area and in Luçon. The analysis of the demographic evolution highlights two peaks: the first in the 1860s and the second in the current period, as well as a sharp increase in the littoral areas. Over a period of 300 years and at a fine spatial scale, our results illustrate national phenomena, such as the great rural exodus and littoralization.
\end{abstract}

Mots clés : Marais maritime - urbanisation - étalement urbain - démographie - pression anthropique - haliotropisme

Abstract: Coastal marsh - urbanization - urban sprawl - demography - littoralisation 


\section{INTRODUCTION}

Le littoral est un espace d'extrême concentration des activités humaines (Halpern et al., 2008). Sur cette bande étroite, les zones humides ${ }^{1}$ ont été très tôt drainées, aménagées et reconverties en terres arables et leurs bordures côtières ont été souvent urbanisées à des fins touristiques. C'est pourtant sur ces espaces littoraux que se concentrent les enjeux de conservation de la biodiversité, aujourd'hui internationalement reconnus (Costanza et al., 1997 ; Gray, 1997 ; Beaumont et al., 2007). Dans ce contexte est apparue la nécessité de ralentir les impacts de cette anthropisation et notamment d'acquérir à de fins conservatoires les derniers espaces peu anthropisés. L'étude de l'évolution historique de l'anthropisation des zones humides est donc essentielle à toute stratégie conservatoire.

Bien qu'encore peu fréquentes, les données concernant les changements d'occupation et d'usage du sol sur le long terme dans les zones humides commencent à voir le jour (Godet et Thomas, 2013). Toutefois, les études mettant en lumière les évolutions historiques de la distribution du bâti et de la démographie à l'échelle de zones humides restent lacunaires. Pourtant, le bâti et la démographie sont bien deux indicateurs clés du degré d'anthropisation de tels espaces. L'urbanisation est une des causes majeures de crise de la biodiversité dans le monde, conduisant à une perte d'habitats naturels, une disparition d'espèces et à une homogénéisation biotique (McKinney, 2002). Plus particulièrement, les zones humides ont été soumises à une urbanisation parfois de grande ampleur (e.g. Bolca et al., 2007), conduisant à leur disparition progressive ou à l'altération de leur fonctionnement (Lee et al., 2006) ainsi qu'à une érosion de biodiversité qui leur est associée (Guzy et al., 2012; Johnson et al., 2013).

L'objectif de cet article est de fournir : (i) une première cartographie fine de l'évolution du bâti d'une portion de zone humide française sur trois cents ans; (ii) une analyse de l'évolution démographique de ce même espace sur deux cents ans. Ces résultats seront ensuite mis en relation avec les changements d'occu-

1. Nous entendons ici par «zones humides » la définition classiquement admise dans la Convention de Ramsar : «Des étendues de marais, de fagnes, de tourbières ou d'eaux naturelles ou artificielles, permanentes ou temporaires, où l'eau est stagnante ou courante, douce, saumâtre ou salée, y compris des étendues d'eau marine dont la profondeur à marée basse n'excède pas six mètres. » pation du sol qui ont affecté cet espace et les changements démographiques de l'ouest français.

\section{MATÉRIEL et MÉTHOdes}

Les zones humides intéressent la recherche depuis longtemps du fait de cette dichotomie entre leurs richesses naturelles et leur anthropisation. Ainsi, Davidson (2014) s'est intéressé aux études historiques des marais dans le monde afin de quantifier leur disparition. Bien que la France ait accusé un certain retard sur cette approche de géographie historique des zones humides (Derex, 2001), le sujet est aujourd'hui abondamment traité. Il est un moyen de connaître l'évolution historique de ces territoires (Valette et Carozza, 2010; Franchomme et Schmitt, 2012) et peut nous éclairer sur une des problématiques actuelles majeures de ces espaces : le risque d'inondation (Antoine et al., 2009; Dournel, 2014) et de submersion marine (Perret et Sauzeau, 2014).

\section{Site d'étude : la partie occidentale du Marais poitevin}

Le Marais poitevin, situé sur la côte atlantique française (figure 1 - planche VII), est la deuxième zone humide de France après la Camargue et couvre environ 100000 ha (Verger, 2005). Il s'agit probablement d'un des meilleurs exemples des bouleversements dans l'usage et l'occupation des sols en zone humide, tant les reconversions des milieux naturels en terres agricoles et la modification du trait de côte au gré des poldérisations (Verger, 1968) y ont été massives (Godet et Thomas, 2013; Godet et al., 2015). Les grands assèchements ont débuté dès le XIII ${ }^{\mathrm{e}}$ siècle (Clouzot, 1904; Sarrazin, 1985), puis surtout au XVII ${ }^{\text {e }}$ siècle (Suire, 2006) et se sont poursuivis aux XIX ${ }^{\mathrm{e}}$ et $\mathrm{XX}^{\mathrm{e}}$ siècles (Billaud, 1984). C'est en grande partie du fait des changements très récents de la fin du $\mathrm{xx}^{\mathrm{e}}$ siècle, que le site est le seul à avoir perdu son label de Parc Naturel Régional (PNR) en 1996, qu'il a récemment retrouvé suite au décret du 21 mai 2014. En 1999, la France est même condamnée par la Cour Européenne de Justice à verser une amende pour son incapacité à maintenir des mesures de protection nécessaires à la conservation des oiseaux et à éviter la dégradation de leurs habitats. Il s'agit en outre de la seule zone humide européenne d'une telle étendue à ne pas 
être incluse dans le réseau RAMSAR (malgré une concentration de ces sites en Europe occidentale voir Frazier, 1999).

Nous avons choisi d'étudier un espace incluant 10 communes de la partie occidentale du Marais poitevin car il offre un gradient spatial partant de la plaine vendéenne au nord (Luçon) jusqu'au littoral au sud (La Faute-sur-Mer, L'Aiguillon-sur-Mer) en passant par les polders de l'Anse de l'Aiguillon et les marais desséchés, où l'on trouve les «îles » calcaires occupées par les communes de Grues, Triaize et SaintMichel-en-l'Herm (qui correspondent à d'anciennes îles du Golfe des Pictons). Cet espace correspond approximativement au périmètre administratif du canton de Luçon, duquel a été exclu la commune de Sainte-Gemme-la-Plaine, située dans la plaine calcaire et donc hors du marais, et en y ajoutant la commune de la Faute-sur-Mer, située sur une flèche littorale et donc sur un ensemble côtier très sensible.

\section{Données d'évolution du bâti}

\section{Matériel cartographique et photographique}

Nous avons utilisé quatre lots de données cartographiques (figure 2). Le premier lot correspond à deux cartes (carrés $n^{\circ} 9$ et $n^{\circ} 13$ ) dressées par Claude Masse (1652-1737), qui sont actuellement stockées au Service Historique de la Défense à Vincennes. Ces cartes ont été levées en 1705 et leur échelle approximative est le 1/28000 (voir Suire, 2011 , pour une description détaillée de ces cartes). Le deuxième jeu de cartes correspond à la carte de l'État-major (feuille « Fontenay-Le-Comte»), disponible en accès libre sur www.geoportail.fr, levées en 1820 sur ce secteur d'après Godet et Thomas (2013) et dont l'échelle est le 1/40000. Le troisième jeu de données concerne les photographies aériennes de mai 1950 (mission "F1229-1629") au 1/26000. Enfin, le quatrième lot de données correspond à la base de données vectorisée des éléments bâtis, disponible auprès de l'IGN (Institut Géographique National) (IGN-BD-TOPOß) de 2006.

\section{Traitement des données cartographiques et photographiques}

Les cartes de 1705 ont été numérisées à une résolution de 400 dpi. Les cartes de 1820 et les photo- graphies aériennes de 1950 étaient déjà numérisées à haute résolution par l'IGN. Les cartes et photographies aériennes ont été géoréférencées et orthorectifiées à l'aide du logiciel Geomatica v.9@ à partir de points de contrôle pris sur l'orthophotographie de l'IGN de 2006 ainsi que sur les cartes au 1/25000 de l'IGN (IGN-BD-ORTHO ${ }^{\circledR}$ et IGN-SCAN-25® respectivement), en sélectionnant les points de repère restés inchangés entre les différentes dates (intersections de routes et canaux et bâtiments historiques principalement). Toutes les données géoréférencées ont été intégrées à un Système d'Information Géographique (SIG) (ESRI@ ArcGis 10.1®).

\section{Cartographie du bâti}

Tous les bâtiments présents sur les cartes de 1705 et 1820 ainsi que sur les photographies de 1950 ont été vectorisés un à un sous SIG (figure 2) et tous les bâtiments de la BD-TOPO ${ }^{\circledR}$ ont été importés (éléments «bâti indifférencié », «bâti industriel » et «bâti remarquable» de la base de données).

Afin de produire une cartographie générale de l'évolution des surfaces bâties aux quatre dates (1705, 1820, 1950, 2006), une maille carrée régulière de 250 m de côté (soit 8754 mailles de 6,25 ha) a été appliquée à la zone d'étude. Une surface totale bâtie au sein de chacune de ces mailles a ensuite été calculée sous SIG.

Les données cartographiques brutes représentant chaque bâtiment à chaque date ont été utilisées pour produire les cartes d'évolution du bâti de trois bourgs : Luçon, Saint-Michel-en-l'Herm et L’Aiguillon-sur-Mer/La Faute-sur-Mer.

\section{Données de recensements démographiques}

Les recensements communaux des 10 communes de 1801 à nos jours ont été utilisés pour évaluer l'évolution démographique des communes de l'espace d'étude (figure 2). La commune de La Fautesur-Mer n'ayant été érigée en commune que le 18 décembre 1953 (à la suite de sa séparation avec la commune de La Tranche-sur-Mer), les premiers recensements utilisés pour cette commune sont de 1954. 


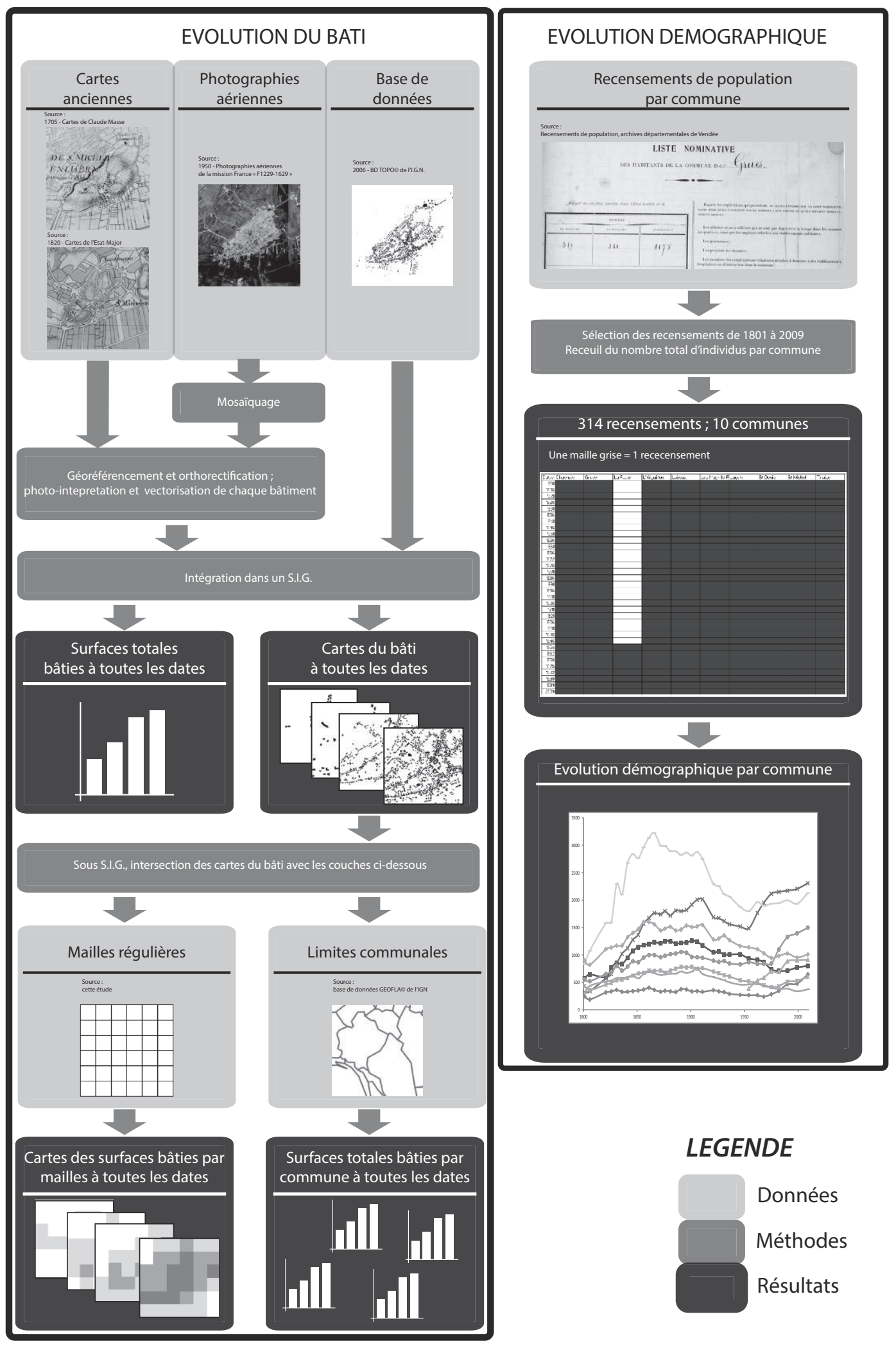

Figure 2 : Méthodologie de cartographie historique du bâti et d'évaluation de l'évolution démographique Methodology used for the historical mapping of the buildings and to assess the demographic evolution 


\section{Résultats}

\section{Distribution du bâti : une évolution constante dans le temps mais inégalement répartie dans l'espace}

À l'échelle de l'ensemble de la zone d'étude, les surfaces bâties sont en constante progression tout au long de la période d'étude selon un modèle de distribution d'habitat très groupé sur la plaine vendéenne, sur les îles, puis, plus tardivement, le long du littoral (figure 3 - planche VIII). En dehors de ces agglomérations, l'habitat reste dispersé et représenté par des fermes isolées aussi bien dans le marais desséché que dans les polders. Trois périodes peuvent être distinguées dans la progression du bâti : une première phase de croissance modérée de 1700 à 1820 (+39,5 ha en 120 ans); une seconde phase de progression plus lente entre 1820 et 1950 (+ 7,8 ha en 130 ans); et enfin une forte augmentation lors des 60 dernières années (+ 193,5 ha) (figure 3 - planche VIII).

Toutefois, ces progressions sont très inégalement distribuées. De 1705 à 1820, l'évolution des surfaces bâties se fait surtout autour des plus gros bourgs, tout particulièrement autour des bourgs de Luçon, et, dans une moindre mesure, des îles de Saint-Michel-en-l'Herm, de Triaize, Grues et de la presquîlle de Saint-Denis-du-Payré. On peut également noter le développement du bâti dès 1950 au sein des actuelles communes de La Faute-sur-Mer et de L'Aiguillon-sur-Mer où le bâti était encore inexistant en 1705 et essentiellement cantonné au cœur du bourg de L'Aiguillon-sur-Mer en 1820. De 1950 à 2006 le bâti progresse toujours au sein des plus gros bourgs et reste très groupé, mais l'évolution la plus spectaculaire concerne la frange littorale de part et d'autre de l'embouchure du Lay à La Faute-sur-Mer et L'Aiguillon-sur-Mer.

Sur les trois communes de Luçon, Saint-Michelen-l'Herm et La Faute-sur-Mer/L’Aiguillon-sur-Mer, on observe trois types différents de progression du bâti (figures 4 à 6).

À Luçon (figure 4- planche IX), le bâti progresse de manière rapide et constante. À partir du noyau historique, le bâti se développe d'abord au sein des espaces interstitiels et le long des principales routes de 1705 à 1820 (+ 12,1 ha en 115 ans). Cette progression se poursuit de 1820 à $1950(+8,6$ ha en 130 ans) à partir des axes routiers. De 1950 à 2006 le bâti progresse très rapidement $(+64,3$ ha en 56 ans), ceci surtout dû à l'implantation de lotissements résidentiels qui viennent combler les espaces encore non bâtis entre les grands axes de communication ainsi qu'au développement de zones industrielles et d'activités en périphérie de la ville. Tout au long de la période d'étude, l'extension du bâti de la ville s'est faite essentiellement sur la plaine calcaire.

À Saint-Michel-en-l'Herm (figure 5-planche IX), le bâti reste circonscrit au périmètre de l'ancienne île. À partir du bourg historique, le bâti se développe autour de ce noyau situé au centre-sud et sur la bordure méridionale et occidentale de l'île de 1705 à 1820 (+ 5,1 ha en 115 ans). De 1820 à 1950, la progression se poursuit principalement dans ces mêmes secteurs et le long des axes nord-est / sud-ouest de l'̂lle (+ 3,4 ha en 130 ans). Enfin, de 1950 à 2006, le reste de lîle est colonisé (+ 22 ha).

Le secteur de L'Aiguillon-sur-Mer/La Faute-surMer offre l'exemple le plus spectaculaire d'évolution du bâti (figure 6 - planche $\mathbf{X}$ ). En 1705, l'amorce de l'actuelle Pointe d'Arçay, sur la rive sud du Lay, est totalement inhabitée et le village de L'Aiguillonsur-Mer, sur la rive nord du Lay, est situé à environ $4 \mathrm{~km}$ au sud-est du village actuel, autour de l'ancien fort de l'Aiguillon, aujourd'hui disparu (Perret et Sauzeau, 2014). En 1820, seules quelques maisons sont présentes sur la rive sud du Lay (approximativement 15 constructions) et sur la rive nord, le village de l'Aiguillon est situé à $4 \mathrm{~km}$ au nord-ouest de sa position initiale de 1705 et les surfaces bâties ont un peu progressé (+2 ha). En 1950, le village de l'Aiguillon est plus étendu de part et d'autre du noyau de 1820 (+ 4,9 ha) et l'actuel bourg de la Faute a également beaucoup progressé $(+2,3$ ha). L'essor du bâti est spectaculaire de 1950 à 2006 : la surface bâtie passe de 10,4 à 38,6 ha à L'Aiguillonsur-Mer et de 3,3 à 36,3 ha à La Faute-sur-Mer. À elles deux, les deux communes ont gagné 61,2 ha en 56 ans, soit presque autant que la commune de Luçon sur la même période $(+64,3$ ha) et trois fois plus que la commune de Saint-Michel-en-l'Herm (+ 22 ha).

\section{Évolution demographique : une évolution en quatre phases}

Dans l'ensemble de la zone d'étude, l'évolution démographique connait quatre étapes (figure 7 - 
planche $\mathbf{X})$. La population augmente d'abord de manière soutenue de 1801 à 1861 (modèle linéaire : $+153,42$ habitants par an $\pm 5,76$ e.s., $\mathrm{R}^{2}=0,99$, $\mathrm{P}<0,0001$ ), puis augmente faiblement durant les cinquante années suivantes de 1861 à 1911 (modèle linéaire : $+37,82$ habitants par an $\pm 3,44$ e.s., $\mathrm{R}^{2}=$ $0,79, \mathrm{P}=0,0002)$. Elle tend à chuter de manière non significative ensuite de 1911 à 1946 (modèle linéaire : $-44,93$ habitants par an $\pm 18,29$ e.s., $\left.\mathrm{R}^{2}=0,50, \mathrm{P}=0,07\right)$ puis progresse significativement de nouveau de 1946 à 2009 (modèle linéaire : $+77,36$ habitants par an $\pm 3,66$ e.s., $\mathrm{R}^{2}=0,98$, $\mathrm{P}<0,0001)$ en atteignant à la fin des années 1970 une population identique à celle des années 1860 . La plupart des communes suit cette évolution démographique en quatre étapes, hormis Luçon, ville la plus importante du site d'étude. Luçon est en effet en progression presque constante tout au long de la période (figure $7 \mathrm{~b}-$ planche $\mathbf{X}$ ). Malgré une période de stagnation à la fin du XIX siècle et une légère baisse de 1906 à 1926, le nombre d'habitants passe de 2632 à 9784 de 1801 à 2009, soit en moyenne un peu plus de 31 habitants supplémentaires par an sur la période considérée (modèle linéaire : + 31,27 habitants par an \pm 1,32 e.s., $\left.\mathrm{R}^{2}=0,94, \mathrm{P}<0,0001\right)$.

On peut également noter que quatre communes ont connu une progression démographique particulièrement importante depuis la fin des années 1960 : L'Aiguillon-sur-Mer et La Faute-sur-Mer sur le littoral et Les Magnils-Reigners et Chasnais immédiatement à l'ouest de Luçon.

\section{Discussion}

\section{Une dynamique d'anthropisation inégalement répartie dans l'espace et le temps}

Les évolutions du bâti et de la démographie peuvent être considérées comme deux indicateurs d'anthropisation d'un espace. À l'issue de cette étude, la première constatation est qu'elles sont toutes deux en progression assez importante pour un territoire rural.

On remarque toutefois une irrégularité à la fois temporelle et spatiale à cette progression. On peut ainsi distinguer quatre phases d'évolution spatiale du bâti et de la démographie qui sont à mettre en relation avec la démographie des campagnes françaises et les changements d'occupation et d'usage des sols de cette zone humide.

Au XIX ${ }^{e}$ siècle, la plupart des communes étudiées ici connaissent d'abord un "plein rural », tel que défini par Dupâquier (1989), c'est-à-dire l'atteinte d'un maximum démographique, aux alentours de 1860. Celui-ci intervient donc un peu plus tardivement que dans le reste de la France, où il a lieu autour de 1850, mais plus précocement qu'à l'échelle du département de la Vendée, où il est estimé à 1896 (Dupâquier, 1989). Le XIx ${ }^{e}$ siècle est également marqué par une progression nette du bâti à l'échelle de toute la zone d'étude par rapport à la situation du début du XVIII ${ }^{\mathrm{e}}$ siècle. Cette progression démographique et du bâti est à mettre en relation avec un essor des cultures sur labour très important au détriment de milieux peu ou pas artificialisés tels que les marécages et les surfaces en prairies (Godet, Thomas, 2013). L'essor démographique se traduit donc logiquement par un essor des surfaces bâties et la main-d'œuvre disponible a permis le développement massif de labours. Le XIX ${ }^{\mathrm{e}}$ siècle est d'ailleurs la période durant laquelle le Marais poitevin (plus particulièrement les marais desséchés) est caractérisé par un système de grandes cultures divisées en vastes propriétés (Billaud, 1984).

Une relative stagnation démographique caractérise ensuite la deuxième moitié du XIX ${ }^{\mathrm{e}}$ siècle jusqu'à la Première Guerre mondiale. À la chute démographique des années 1910 jusqu'au lendemain de la Seconde Guerre mondiale correspond une progression assez lente des surfaces bâties de 1820 à 1950, surtout en faveur des espaces déjà agglomérées. Durant cette période d'exode rural en France, seule la principale agglomération de la zone d'étude, Luçon, progresse démographiquement. Cette période correspond également à un morcellement de la propriété agricole (Billaud, 1984) et à une progression spectaculaire des surfaces en prairie au détriment des surfaces labourées (Godet et Thomas, 2013). Quoique moins important que durant la première moitié du XIX ${ }^{\mathrm{e}}$ siècle, l'essor démographique est important depuis les années 1950. On peut distinguer quatre tendances différentes sur cette dernière période.

Tout d'abord, Luçon connait une augmentation importante de sa population et un étalement urbain significatif sur la période. C'est le pôle d'attraction 
économique du secteur puisqu'il concentre $71 \%$ des emplois de la zone d'étude (tableau 1, INSEE, 2011). En 2011, la commune compte près de deux fois plus d'emplois que d'actifs disponibles dans la commune (ratio de 0,57, tableau 1). Ce déficit est comblé par les migrations pendulaires des communes voisines.

Ainsi, les communes périphériques progressent également, mais dans une moindre mesure. Ceci s'explique principalement par le phénomène de périurbanisation rendu possible par le développement des migrations pendulaires grâce aux modes de transports individuels (François, 2010). La population active de ces communes travaille pour partie à Luçon (OESTV, 2007). Ces communes ont toutes une population active occupant un emploi supérieure au nombre d'emplois effectivement disponibles sur la commune. Cela est particulièrement frappant aux Magnils-Régniers où la population active travaillant est quatre fois supérieure aux nombre d'emplois qui y sont effectivement disponibles (ratio de 3,90, tableau 1).

L'exemple de Saint-Michel-en-l'Herm montre une meilleure adéquation entre la population active et le nombre d'emplois (tableau 1), ce qui démontre une moindre influence du pouvoir d'attraction de Luçon.

Enfin, cette période se caractérise également par une forte littoralisation du bâti et un essor démographique des deux communes littorales de l'Aiguillon-sur-Mer et de la Faute-sur-Mer, ce qui n'est pas sans poser des problèmes de gestion ou d'ordre environnemental (Renard, 2005 ; Chadenas et al., 2013, Creach et al., 2015). Ceci est la traduction d'un développement centré sur l'activité touristique et balnéaire. Il repose sur l'accueil de résidents non permanents. Ainsi, la commune de La Faute-
sur-Mer a connu une multiplication par sept du nombre de logements entre 1950 et 2010, tandis que la population permanente a été multipliée par trois dans le même laps de temps (INSEE, 2011; Vinet et al., 2012). Cela se traduit donc par une très forte représentation des résidences secondaires. En 2011, la commune comptait $85 \%$ de logements occupés de façon non permanente (INSEE, 2011). Cette répartition n'est pas nouvelle puisqu'en 1968 on dénombrait déjà $79 \%$ de résidences secondaires. L’Aiguillon-sur-Mer présente un bilan plus équilibré avec $42 \%$ de résidences secondaires en 2011 (INSEE, 2011). Ces deux profils dénotent à l'échelle cantonale où, en dehors de La Faute-surMer, on compte $78 \%$ de résidences principales pour $22 \%$ de résidences secondaires. Enfin, on remarque que ces communes, dont l'activité économique est saisonnière, présentent un déficit de main-d’œuvre (ratio de 0,77 - tableau 1).

\section{Les limites inhérentes à l'objet d'étude}

Les premiers résultats présentés ici doivent néanmoins être quelque peu nuancés. En effet, nous nous basons sur la numérisation de la géométrie des constructions pour estimer les surfaces bâties au cours du temps. Pour cela, nous avons utilisé quatre sources de données différentes. Nous aurions pu estimer le nombre de constructions, en plus des surfaces urbanisées. Néanmoins, cela ne nous était pas possible dans les zones densément urbanisées. La mitoyenneté des constructions dans ces secteurs rend délicate l'estimation du nombre de constructions individualisées pour les années 1705, 1820 et 1950. Les données issues de la BD-TOPO $\AA$ permettent, par contre, de distinguer les constructions

\begin{tabular}{|c|c|c|c|c|c|c|c|c|c|}
\hline \multirow{2}{*}{ RGP 2011} & \multirow[t]{2}{*}{ Canton } & \multicolumn{2}{|c|}{ Luçon } & \multicolumn{2}{|c|}{$\begin{array}{l}\text { Les Magnils- } \\
\text { Reigniers }\end{array}$} & \multicolumn{2}{|c|}{$\begin{array}{l}\text { Saint-Michel- } \\
\text { en-l'Herm }\end{array}$} & \multicolumn{2}{|c|}{ L'Aiguillon-sur-Mer } \\
\hline & & & $\%$ cant. & & $\%$ cant. & & $\%$ cant. & & $\%$ cant. \\
\hline Population totale & 19813 & 9536 & $48,1 \%$ & 1508 & $7,6 \%$ & 2235 & $11,3 \%$ & 2261 & $11,4 \%$ \\
\hline Nombre d'actifs travaillant & 6736 & 3314 & $49,2 \%$ & 687 & $10,2 \%$ & 693 & $10,3 \%$ & 522 & $7,7 \%$ \\
\hline Nombre d'emplois & 8198 & 5829 & $71,1 \%$ & 176 & $2,1 \%$ & 567 & $6,9 \%$ & 678 & $8,3 \%$ \\
\hline $\begin{array}{l}\text { \% du nombre d'actifs travaillant/ } \\
\text { population totale }\end{array}$ & $34,0 \%$ & \multicolumn{2}{|c|}{$34,8 \%$} & \multicolumn{2}{|c|}{$45,6 \%$} & \multicolumn{2}{|c|}{$31,0 \%$} & \multicolumn{2}{|c|}{$23,1 \%$} \\
\hline $\begin{array}{l}\text { Ratio du nombre d'actifs travail- } \\
\text { lant/nombre d'emplois }\end{array}$ & 0,82 & \multicolumn{2}{|c|}{0,57} & \multicolumn{2}{|c|}{3,90} & \multicolumn{2}{|c|}{1,22} & \multicolumn{2}{|c|}{0,77} \\
\hline
\end{tabular}

Tableau 1 : Statistiques de l'emploi sur quatre communes de la zone d'étude Employment statistics in four towns of the study area 
à l'intérieur d'îlots agglomérés. Il serait néanmoins hasardeux d'extrapoler à partir de cette donnée le nombre de constructions pour les dates antérieures.

La concordance des résolutions spatiales pour valider des changements d'occupation du sol est souvent déterminante (Bousquet et al., 2013). Le calcul des surfaces bâties repose sur la précision des données utilisées et sur la qualité du géoréférencement. La précision des données utilisées est évidemment variable, même si dans le cas présent les documents sont d'une très grande qualité. La précision de ces données s'exprime par exemple dans leurs échelles différentes. La représentation du bâti sur les cartes de l'État-major s'avère être plus grossière (échelle du 1/40 000) que celle des cartes de Claude Masse ou que celle des photos aériennes (échelles proches du 1/27 000). La précision des géoréférencements peut également être à l'origine de décalages et d'erreurs dans le calcul des surfaces. C'est notamment pour pouvoir lisser ces différences de résolution que nous avons présenté des cartes d'évolution moyennées sur un carroyage de 250 mètres de côté.

Dans une perspective d'étude de l'urbanisation dans le temps et l'espace il serait riche d'enseignement d'avoir des informations sur l'ancienneté du bâti actuel à l'échelle du bâtiment lui-même. Les cartes que nous proposons ici sur quelques communes ne sont que des superpositions graphiques du bâti à plusieurs dates mais elles ne nous renseignent pas sur l'ancienneté de chaque bâtiment. Le résumé de trois cents ans d'évolution du bâti condensé en quatre dates tel que proposé dans cette étude masque en effet de possibles allers et retours et renouvellements dans la progression du bâti dans le temps.

Enfin, les limites communales utilisées sont basées sur la base de données GEOFLA. Le contour des communes est parfois très généralisé et a pu conduire à quelques erreurs d'attribution de surfaces bâties d'une commune à une autre.

\section{Perspectives : vers une extension spatiale et temporelle?}

Cette étude apporte des premiers chiffres sur la dynamique du bâti dans la partie occidentale du Marais poitevin. Nous nous sommes focalisés sur dix communes correspondant approximativement au territoire du canton de Luçon. Cette zone offre une première approche intéressante par ses divers faciès (littoral, polder, "îles », plaine, présence d'une ville de taille moyenne). Néanmoins, il pourrait être intéressant d'étendre ce travail à l'ensemble du marais. La partie charentaise du Marais poitevin présente des différences sensibles avec la zone que nous étudions ici. Ainsi, les communes littorales en Charente-Maritime connaissent un développement différent de celui observé à La Faute-sur-Mer et à L'Aiguillon-sur-Mer, notamment concernant la composition de la population (Vinet et al., 2012). Alors que ces deux dernières connaissent une proportion de résidents secondaires proche des $80 \%$, les communes de Charron et Esnandes, du fait de leur proximité à La Rochelle, accueillent surtout des résidents principaux. Le développement de l'urbanisation y est donc légèrement plus limité que dans la partie vendéenne du marais.

La partie orientale du marais présente des caractéristiques encore différentes puisqu'elle ne possède pas de façade littorale. La dynamique d'urbanisation y est probablement plus proche de la partie centrale de notre zone d'étude.

Une extension temporelle de l'étude serait également pertinente compte tenu des très récents changements qu'a connu le bâti des communes de La Faute-sur-Mer et de L'Aiguillon-sur-Mer suite à la tempête Xynthia et aux démolitions de nombreuses maisons.

La remarquable qualité des archives des recensements de la population rendrait intéressant un travail complémentaire sur ces données. En plus de connaître la composition de la population communale, on y trouve des informations sur « le titre, la qualification, la profession ou fonction » des individus, ainsi que leur adresse. Il serait par exemple possible de confirmer le passage d'une société agraire composée de quelques riches propriétaires terriens et nombreux ouvriers agricoles vers une société de services avec de nombreuses personnes qui ne travaillent pas dans leur commune de résidence.

\section{Conclusion}

Malgré la connaissance des principaux changements d'occupation du sol en Marais poitevin (Godet et Thomas, 2013), il manquait à ce jour des premières estimations quantifiées et spatialisées de l'implantation humaine dans ce marais au cours des 
derniers siècles. Cette étude apporte les premiers éléments dans la compréhension de l'urbanisation de la partie ouest du marais. À travers la cartographie des surfaces bâties entre 1705 et 2006 et l'analyse des données de population, les premiers résultats illustrent les grandes tendances observées à l'échelle nationale lors des trois cents dernières années. On y observe une première période d'augmentation de la population et des surfaces bâties correspondant au plein rural (du début du XVIII ${ }^{\mathrm{e}}$ siècle à la deuxième moitié du XIX ${ }^{e}$ siècle), suivi d'une période de stagnation (hormis la commune de Luçon) qui correspond à la période d'exode rural (première moitié du $\mathrm{xx}^{\mathrm{e}}$ siècle), et enfin une période d'étalement urbain dans la dernière moitié du $\mathrm{xx}^{\mathrm{e}}$ siècle qui a bénéficié à l'ensemble des communes étudiées et plus particulièrement à la commune de Luçon et aux communes littorales. Ces dernières ont connu un fort essor de leur urbanisation dans la période récente (1950-2006), ce qui illustre le phénomène de littoralisation des activités.

\section{Remerciements}

Nous remercions Laurent Couderchet et un relecteur anonyme pour leurs critiques constructives sur une première version de cet article.

\section{Bibliographie}

Antoine J.-M., Desailly B., Peltier A., 2009. Sources historiques et problématiques de recherche en géographie des risques naturels, Géocarrefour, vol. 84, n 4, p. 229-239.

Beaumont N.J., Austen M.C., Atkins J.P., Burdon D., Degraer S., Dentinho T.P., Derous S., Holm P., Horton T., van Ierland E., Marboe A.H., Starkey D.J., Townsend M., Zarzycki T., 2007. Identification, definition and quantification of goods and services provided by marine biodiversity: Implications for the ecosystem approach, Marine Pollution Bulletin, vol. 54, n³ 3, p. 253-265. DOI:10.1016/j. marpolbul.2006.12.003.

Billaud J.-P., 1984. Marais poitevin une rencontre de la terre et de l'eau, Paris, L'Harmattan, 265 p.

Bolca M., Turkyilmaz B., Kurucu Y., Altinbas U., Esetlili M.T, Gulgun B., 2007. Determination of Impact of Urbanization on Agricultural Land and Wetland Land Use in Balçovas' Delta by Remote Sensing and GIS Technique, Environmental Monitoring and Assessment, vol. 131, p. 409419. DOI: 10.1007/s10661-006-9486-0
Bousquet A., Couderchet L., Gassiat A., Hautdidier B., 2013. Les résolutions des données « occupation du sol» et la mesure changement. Articuler le temps et le thème, L'Espace géogaphique, vol. 42, n 2013-1, p. 61-76.

Chadenas C., Creach A., Mercier D., 2013. The impact of storm Xynthia in 2010 on coastal flood prevention policy in France, Journal of Coastal Conservation, vol. 18, $\mathrm{n}^{\circ} 5$, p. 529-538. doi: 10.1007/s1 1852-013-0299-3

Clouzot E., 1904. Les Marais de la Sère Niortaise et du Lay $d u X^{e}$ à la fin $d u X V I^{e}$ siècle, Champion, Paris et Clouzot, Niort, 282 p.

Costanza R., D’Arge R., Groot R.D., Farber S., Grasso M., Hannon B., Limburg K., Naeem S., O’Neill R.V., Paruelo J., Raskin R.G., Sutton P., Van Den Belt M., 1997. The value of the world's ecosystem services and natural capital, Nature, vol. 387, p. 253-260. doi:10.1038/387253a0

Creach A., Pardo S., Guillotreau P., Mercier D. 2015. The use of a micro-scale index to identify potential death risk areas due to coastal flood surges: lessons from Storm Xynthia on the French Atlantic coast, Natural Hazards, doi: 10.1007/s11069-015-1669-y (sous presse).

Davidson N.C., 2014. How much wetland has the world lost? Long-term and recent trends in global wetland area, Marine and Freshwater Research, vol. 65, n 10, p. 934-941. DOI: $10.1071 / \mathrm{MF} 14173$

Derex J.-M., 2001. Pour une histoire des zones humides en France $\left(\mathrm{xVII}^{\mathrm{e}}-\mathrm{XIX}{ }^{\mathrm{e}}\right.$ siècle). Histoire E Sociétés Rurales, vol. 15, $\mathrm{n}^{\circ} 1$, p. 11-36.

Dournel S., 2014. Géohistoire du risque d'inondation dans les villes du Val de Loire (Nevers, Orléans, Blois, Tours, Angers) : de l'analyse paysagère a la gestion territorialisée de la prévention. Etude postdoctorale, Université d'Orléans, $142 \mathrm{p}$.

DupÂquier J., 1989. Le plein rural en France, Espaces, Populations, Sociétés, vol. 7, n³, p. 349-356.

Franchomme M., Schmitt G., 2012. Les zones humides dans le Nord vues à travers le cadastre napoléonien : les Systèmes d'Informations Géographiques comme outil d'analyse, Revue du Nord, vol. 396, n³ 3, p. 661-680.

François D., 2010. Se rendre au travail : distances et temps de transport s'allongent, La Revue du CGDD, nº spécial, p. 83-98.

Frazier S., 1999. Ramsar sites overview: a synopsis of the world's wetlands of international importance, Wetlands International, Wageningen, $48 \mathrm{p}$.

Godet L., Pourinet L., Joyeux E., Verger F., 2015. Dynamique spatiale et usage des schorres de l'Anse de l'Aiguillon de 1705 à nos jours. Enjeux de conservation d'un patrimione naturel littoral marin. Cybergeo : European Journal of Geography (sous presse).

Godet L., Thomas A., 2013. Three centuries of land cover changes in the largest French Atlantic wetland provide new insights for wetland conservation, Applied Geography, vol. 42, p. 133-139. DOI:10.1016/j.apgeog.2013.05.011

Gray J. S., 1997. Marine biodiversity: patterns, threats and conservation needs, Biodiversity and Conservation, vol. 6, $\mathrm{n}^{\circ}$ 1, p. 153-175. DOI: 10.1023/A:1018335901847 
Guzy J.C., McCoy E.D., Deyle A.C., Gonzalez S.M., HalsTEAD N., Mushinsky H.R. 2012. Urbanization interferes with the use of amphibians as indicators of ecological integrity of wetlands, Journal of Applied Ecology, vol. 49, p. 941952. DOI: 10.1111/j.1365-2664.2012.02172.x

Halpern B.S., Walbridge S., Selkoe K.A., Kappel C.V., Micheli F., D’Agrosa C., Bruno J.F., Casey K.S., Ebert C., Fox H.E., Fujita R., Heinemann D., Lenihan H.S., Madin E.M.P., Perry M.T., Selig E.R., Spalding M., SteNeCK R., Watson R., 2008. A global map of human impact on marine ecosystems, Science, vol. 319, n 5865 , p. 948952. DOI: 10.1126/science. 1149345

INSEE, 2011. Résultats du recensement de la population 2011. Disponible sur: [http://www.insee.fr/fr/bases-de-donnees/ default.asp?page=recensement/resultats/2011/rp2011.htm].

Johnson P.T.J., Hoverman J.T., McKenzie V.J., Blaustein A.R., Richgels L.D., 2013. Urbanization and wetland communities: applying metacommunity theory to understand the local and landscape effects, Journal of Applied Ecology, vol. 50, p. 34-42. DOI: 10.1111/1365-2664.12022

Lee S.Y., Dunn R.J.K., Young R.A., Connolly R.M., Dale P.E.R., Dehayr R., Lemckert C.J., McKinnon S., Powell B., Teasdale P.R., Welsh D.T., 2006. Impact of urbanization on coastal wetland structure and function, Austral Ecology, vol. 31, p. 149-163. DOI: 10.1111/j.14429993.2006.01581.x

McKinney M.L., 2002. Urbanization, biodiversity, and conservation, BioScience, vol. 52, n 10, p. 883-890. DOI: http:// dx.doi.org/10.1641/0006-3568(2002)052[0883:UBAC]2.0 $\mathrm{CO} ; 2$.

OESTV (Observatoire Économique Social et Territorial de la Vendée), 2007, Les migrations domicile-travail en Vendée en 2007, 6 p.
Perret J., Sauzeau T., 2014. Xynthia ou la mémoire réveillée. Des villages charentais et vendéens face à l'océan (XVIII ${ }^{e}$ XXI ${ }^{e}$ siècles), La Crèche, Geste, 296 p.

Renard J., 2005. La Vendée : un demi-siècle d'observation d'un géographe, Rennes, PUR, 308 p.

SARrazin J.-L., 1985. Maîtrise de l'eau et société en marais poitevin (vers 1150-1283), Annales de Bretagne et des pays de l'Ouest, vol. 92, n 4, p. 333-354.

Suire Y., 2006. Le Marais poitevin: Une écohistoire du XVI à l'aube $d u \mathrm{XX}^{e}$ siècle, Centre Vendéen de Recherches Historiques, La Roche-sur-Yon, 536 p.

Suire Y., 2011. La côte et les marais du Bas Poitou vers 1700, Centre Vendéen de Recherches Historiques, La Roche-surYon, 360 p.

Valette P., Carozza J.-M., 2010. Mise en œuvre d'une démarche géohistorique pour la connaissance de l'évolution des paysages fluviaux : l'exemple de la moyenne vallée de la Garonne. Géocarrefour, vol. 85, n 1, p. 17-27.

Verger F., 1968. Les marais des côtes françaises de l'Atlantique et de la Manche et leurs marges maritimes. Thèse de doctorat ès lettres, Université de Paris, Biscaye Frère Imprimeurs, Bordeaux, $541 \mathrm{p}$.

Verger F., 2005. Marais et estuaires du littoral français, Belin, Paris, $335 \mathrm{p}$.

Vinet F., Defossez S., Rey T., Boissier, L., 2012. Le processus de production du risque "submersion marine » en zone littorale : l'exemple des territoires «Xynthia », Norois, vol. 222, nº 1, p. 11-26. DOI : 10.4000/norois.3834 
planche VII (Pierre Pouzet, Axel CREACH, Laurent GODET - Dynamique de la démographie et du bâti...)

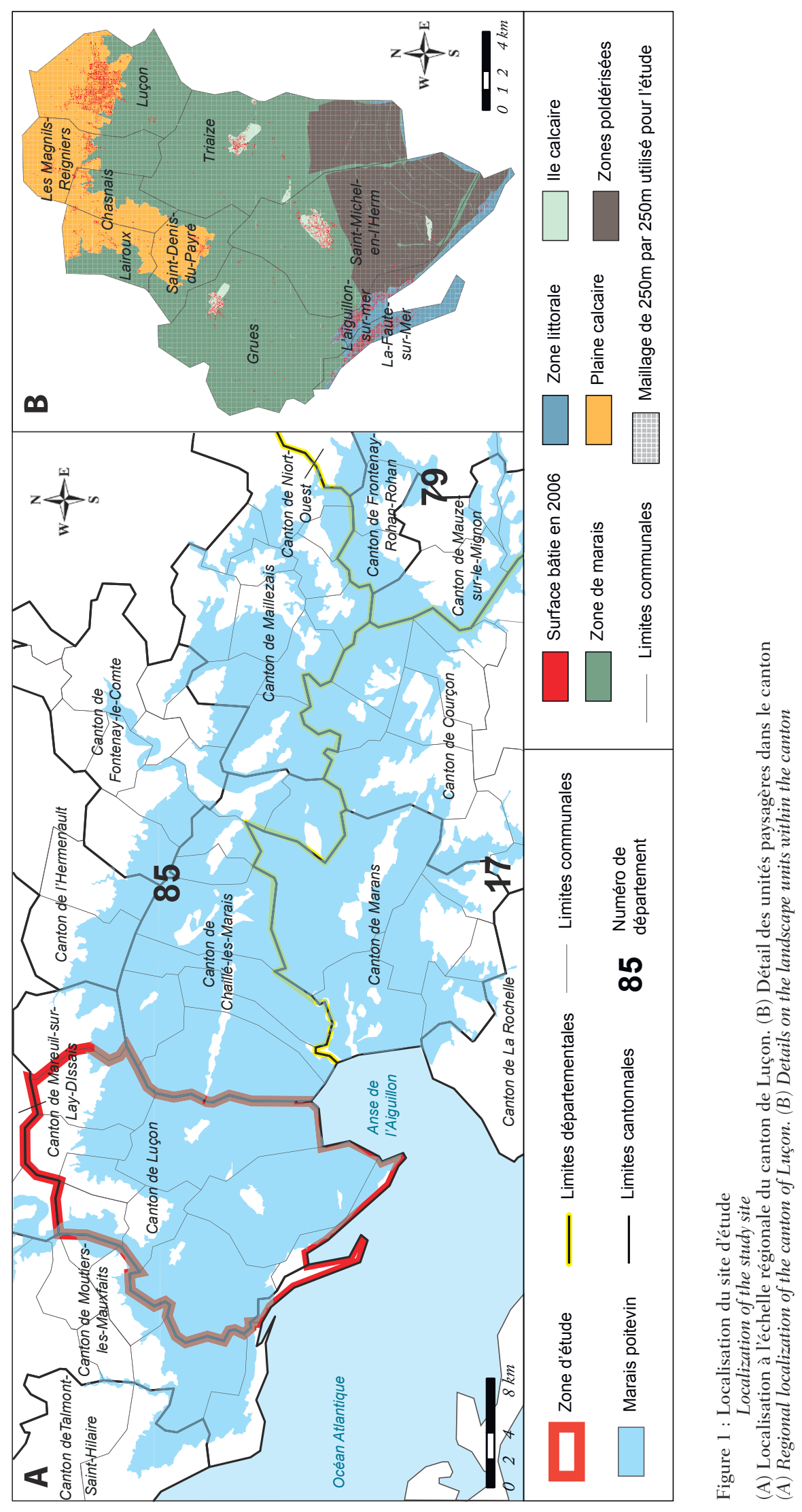



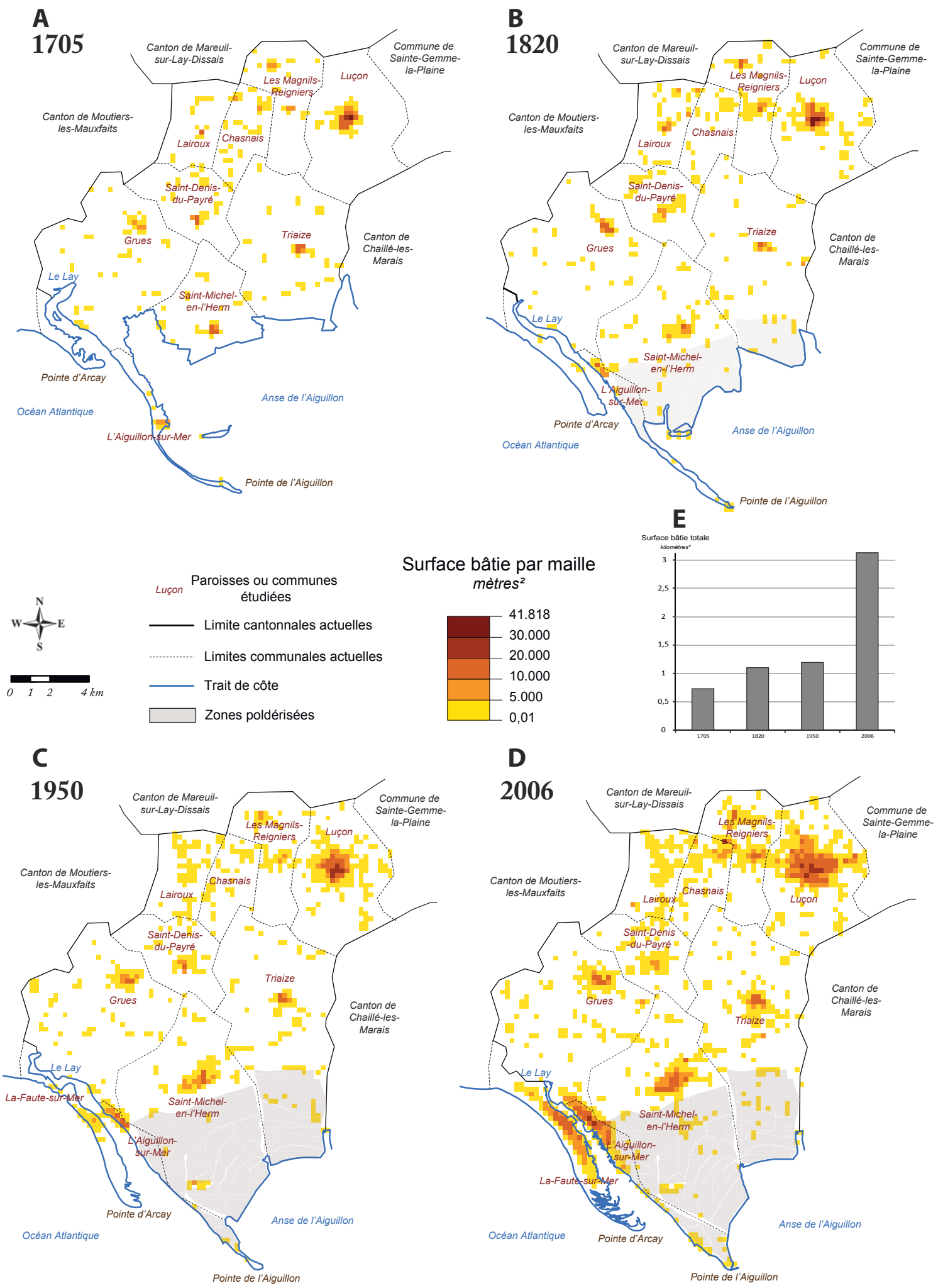

Figure 3 : Évolution de la distribution spatiale des surfaces bâties de 1705 à 2006 Evolution of building distribution from 1705 to 2006

(A) Distribution en 1705. (B) Distribution en 1820. (C) Distribution en 1950. (D) Distribution en 2006. (E) Évolution quantitative. (A) Distribution in 1705. (B) Distribution in 1820. (C) Distribution in 1950. (D) Distribution i 2006. (E) Quantitative evolution. 
planche IX (Pierre Pouzet, Axel CREACH, Laurent GODET - Dynamique de la démographie et du bâti...)
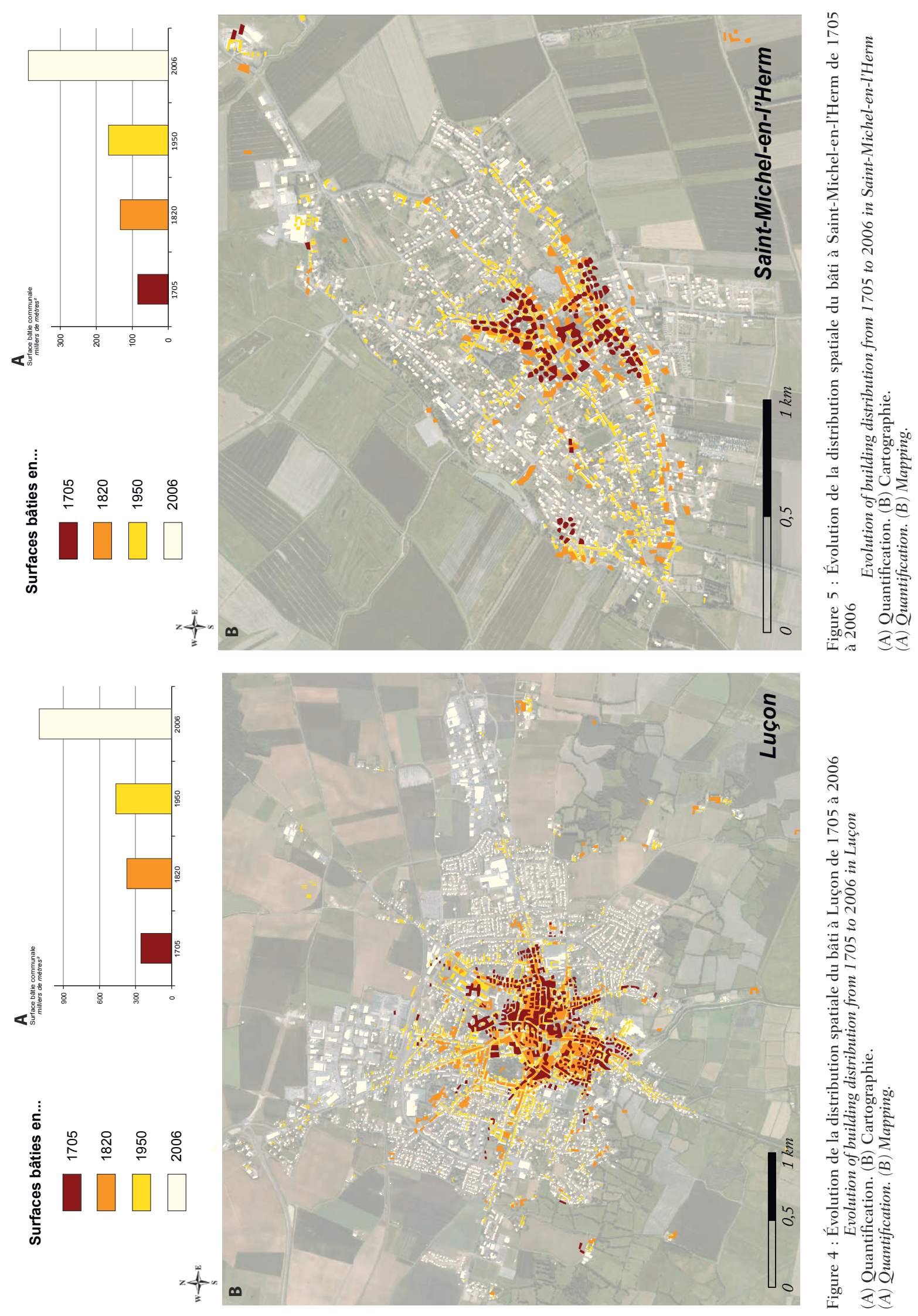

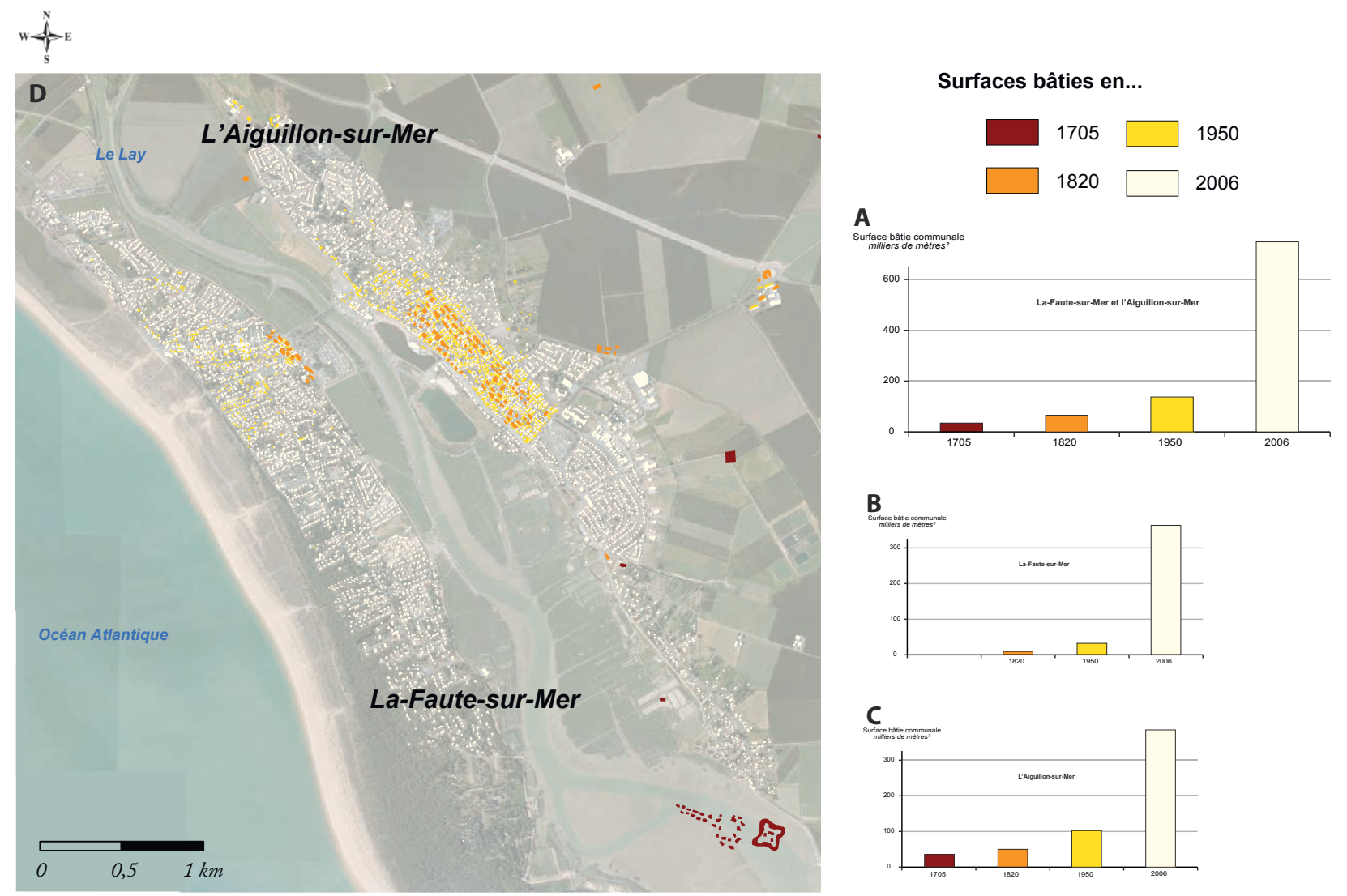

Figure 6 : Évolution de la distribution spatiale du bâti à L'Aiguillon-sur-Mer et La Faute-sur-Mer de 1705 à 2006 Evolution of building distribution from 1705 to 2006 in L'Aiguillon-sur-Mer and La Faute-sur-Mer

(A) Quantification sur les deux communes. (B) Quantification sur La Faute-sur-Mer. (C) Quantification sur L'Aiguillon-sur-Mer. (D) Cartographie. L'ancien centre historique de L'Aiguillon-sur-Mer a également été pris en compte bien qu'il sorte du contour communal actuel. (A) Quantification in the two towns. (B) Quantification in la Faute-sur-Mer. (C) Quantification in L'Aiguillon-sur-Mer. (D) Mapping. Tough located out of the current town perimeter of L'Aiguillon-sur-Mer, its historical inner city was taken into account

\section{A}

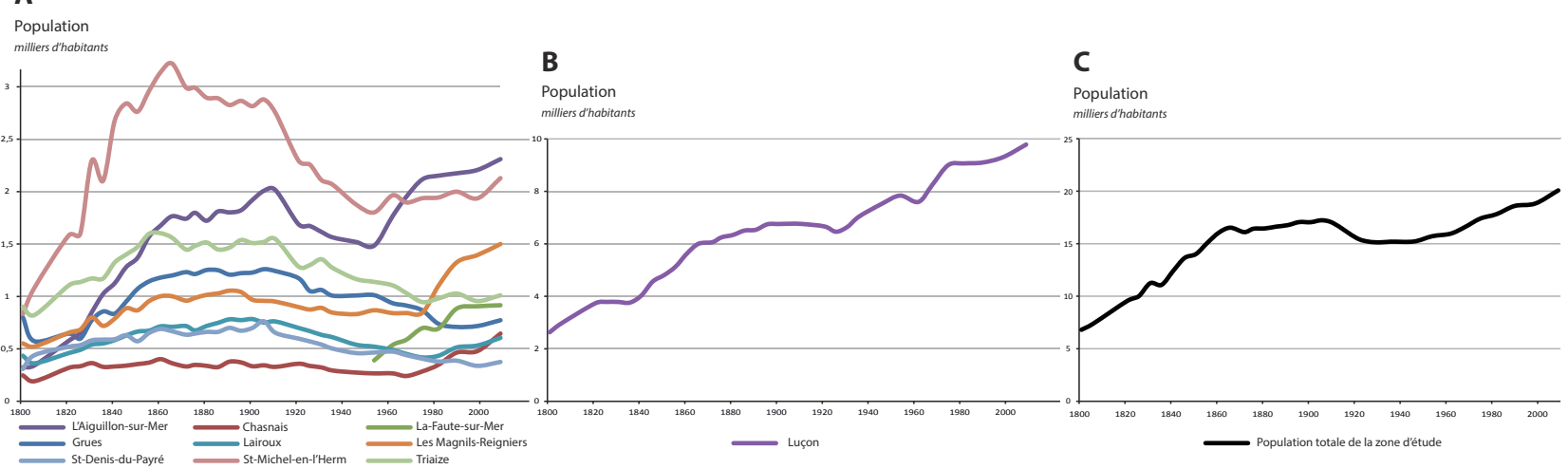

Figure 7 : Évolution démographique des communes de la zone d'étude Demographic evolution of the studied towns.

(A) Toutes les communes hormis Luçon. (B) Commune de Luçon seule. (C) Toutes les communes confondues.

(A) All the towns except Luçon. (B) Luçon only. (C) All the towns. 